\title{
An Overview of SMPC Research Programs to Improve Remelt Ingot Quality
}

\author{
Rodney L. Williamson \\ Remelting Technologies Consulting \\ 12725 Eastridge Trail N.E. \\ Albuquerque, New Mexico 87112, USA
}

Keywords: VAR, ESR, controls, monitors

\begin{abstract}
Research conducted by the Specialty Metals Processing Consortium has focused on developing tools to improve the quality of specialty alloy ingots produced by vacuum arc remelting (VAR) and electroslag remelting (ESR). Especially during the last ten years, the program has focused on developing model-based process monitors and controllers. These tools employ predictive, dynamic, low-order electrode melting and ingot solidification models to estimate important process variables. Process monitors log these estimated variables (some of which are not subject to measurement) to computer files and use them to evaluate the health of the processes. Monitors are capable of detecting and flagging various process upsets and sensor failures. Process controllers use the model-based variable estimates for feedback. Improved melt rate controllers have been developed using this method. Additionally, VAR pool power and pool depth controllers have also been demonstrated. Laboratory and industrial trials have shown the robustness and accuracy of these tools.
\end{abstract}

\section{Introduction}

The Specialty Metals Processing Consortium (SMPC) was formed in 1989 as a vehicle for performing pre-competitive research in the field of specialty metals processing. The consortium consists of specialty alloy producers as well as end users [1], and co-sponsors research with universities and government laboratories. Process specific research has been carried out for vacuum arc remelting (VAR), electroslag remelting (ESR), and electron beam cold hearth melting (EBCHM). In recent years the scope of activities has been narrowed to VAR and ESR. Current research is centered on developing advanced, model-based, discrete-time process monitors and controllers for these two processes in an effort to move toward producing ingots that are free of solidification defects.

The approach taken by the SMPC is to first build process monitors and then, after the monitors have been proven accurate and reliable through industrial testing and application, convert them to process controllers. The monitors developed by the SMPC are discrete time monitors that use discrete-time Kalman filter technology [2]. A Kalman filter is a linear process observer that contains a dynamic process model relating process inputs to outputs. The output of the filter is a prediction of the state of the process at the end of the next time step. The state of the process is defined by a set of values associated with a set of pre-defined process variables. Once the initial state is defined, it can be propagated forward in time using the Kalman filter. Measurements, if available, are used in the filter to correct the model-based predictions. In this way, the monitor may be used to continually track the process and evaluate its overall health. 
One advantage of using this type of process monitor is that it allows the process engineer to make a step-by-step comparison of what should be happening in the process (model-based estimates) with what is actually happening (measurements). The process model represents our best scientific understanding of how the process works under normal conditions. If measurements do not agree with predicted values after taking into account process and measurement uncertainties and model accuracy, then the process is experiencing an upset. Upsets are detected and logged by the monitor to facilitate correlating process conditions with ingot defects and anomalies that appear in post-process inspection and application.

A second advantage of Kalman filter based process monitoring is that the filter can provide estimates of process variables that either cannot be measured or cannot be practically measured. Examples are the temperature distribution in the electrode, electrode/ingot area ratio or fill ratio, electrode gap (VAR), and immersion depth (ESR). Clearly these are important process variables that are unavailable as measured process outputs, but they can be easily estimated using a process observer. For example, when remelting a cast electrode produced using vacuum induction melting, the electrode will have a shrinkage cavity ("pipe") down its center. The diameter and length of the cavity can vary significantly from electrode to electrode. A heavily piped electrode will yield a smaller than nominal fill ratio which can be estimated using the Kalman filter based monitor. This type of process monitoring facilitates finding and logging anomalous process conditions that normally go undetected and which directly impact ingot quality.

Once a process monitor has been proven, it may be converted into a process controller using the Kalman filter output as feedback. This can be done in a variety of ways as described in the references cited below. The advantage is direct control of variables that cannot be measured or practically measured. For example, melt shops have traditionally been interested in controlling the melting rate of the electrode in both VAR and ESR because of its direct impact on ingot quality. However, there is no direct, usable measurement of melt rate. Given current state-of-theart load cell technology and data filtering techniques, step-by-step melt rate measurements are so noisy that they are useless except for averaging over relatively long periods of time. Thus, there is no way to know what the instantaneous melt rate is using weight data alone; one can only know what the average melt rate has been over, say, the last five minutes (or longer). But Kalman filter technology can be used to estimate instantaneous melt rate and accurate, highly dynamic VAR [3] and ESR [4] melt rate controllers have been designed and tested using this technology. This allows for very precisely controlled startup and hot-top procedures based on melt rate which facilitates producing more useable ingot and minimizing the rate of solidification defect formation in these highly transient parts of the remelting process.

In the body of this paper, I will give an overview of the process monitors and controllers developed by the SMPC over the past few years and their impact on ingot quality. Though the examples given will be for VAR, the same principles apply to ESR. The development of both VAR and ESR monitors and controllers has occurred in parallel SMPC programs. Some details will necessarily be missing because of the proprietary nature of the technology. 


\section{SMPC Process Monitors}

Effective process monitoring requires that a single point be located in a multivariable space that characterizes the current state of the process and a determination as to whether that point lies within a region where theory and experience predict successful operation. To facilitate tracking the position of the process in its variable space, a process monitor should be capable of distinguishing between process upsets and sensor noise, as well as detect and report sensor failures. Ideally the monitor would have the additional capability of responding to a failed sensor in a manner that allows it to continue to effectively track the process.

As mentioned above, SMPC process monitors are based on a Kalman filter technology. A Kalman filter produces consistent, unbiased, minimum variance estimates of all state variables associated with the process. The filter used for the VAR process uses a low-order model of electrode melting to provide estimates of the electrode thermal boundary layer $(\Delta)$, electrode gap $(G)$, electrode position $(X)$, electrode weight $(M)$, process efficiency $(\mu)$, fill ratio parameter $(a)$, voltage bias $\left(V_{b}\right)$, current bias $\left(I_{b}\right)$, and electrode velocity bias $\left(U_{b}\right) . \Delta$ is defined as the distance from the electrode tip to that point on the electrode axis where the temperature has reached ambient, and the fill ratio parameter is defined as one minus the ratio of the electrode crosssectional area to that of the crucible $\left(a=1-A_{e} / A_{c}\right)$. The voltage bias is the difference between the estimated process voltage and its measured value; it has proved effective at detecting glows and air and water leaks. Current and ram velocity bias states are necessary from a practical point of view: they account for the fact that the actual current and ram velocity do not quite match the commanded setpoint values in many VAR shops. All bias states are treated as disturbance variables in this formulation. A full treatment of the mathematical development underlying the estimator (Kalman filter) has been reported elsewhere [5] along with a fuller description of the SMPC VAR monitor [6].

Figure 1 shows the SMPC VAR monitor display window [7]. Furnace outputs are displayed in the upper strip-chart control field and Kalman filter (estimator) outputs are displayed in the lower field. The data shown are from an Alloy 718 VAR test melt during steady-state operation. The instantaneous values of the process variables listed along the left side of the window do not represent standard steady-state operating conditions for any SMPC member company.

Besides using estimator outputs to hunt for process upsets and anomalies, the monitor also employs a suite of input data tests that are useful for this purpose. For example, the information listed on the right side of the monitor window indicates that the software has detected two arc shorts (low voltage situations) and two arc-outs (loss of current) in the test data. These two data tests are listed under Standard Tests along with four others: scrams (or electrode pull-out), pressure spikes, glows and helium drop-out (low He pressure) tests. At the conclusion of melting, a report is generated listing the type and location of each event. Other input data tests include Setpoint Deviation Tests, Means Deviations Tests, and Estimator Means Deviation Tests. The Setpoint Deviation Tests check to see if the differences between the values for a furnace setpoint variable and its associated measured values exceed a specified threshold for a specified period of time steps in the test range. For example, the test may be set up to detect when the measured and commanded current differ by more than $1 \%$. The Means Deviations Tests check to see that mean values for all selected furnace variables in the test range fall within the specified deviation limits from the specified targets. For example, the user may want to check that the 
average melt rate over the test range is within $\pm 5 \%$ of the target melt rate. Finally, the Estimator Means Deviation Tests check to see that mean values for all selected estimated furnace variables in the test range fall within the specified deviation limits from the specified targets. For example, the user may want to check that the average efficiency over the test range is within $\pm 1 \%$ of the target efficiency. Note that all test parameters are user-settable in the monitor setup window (not shown). Other than the test variables mentioned above, a comprehensive list of variables available for means deviations tests will not be given because of their proprietary nature.

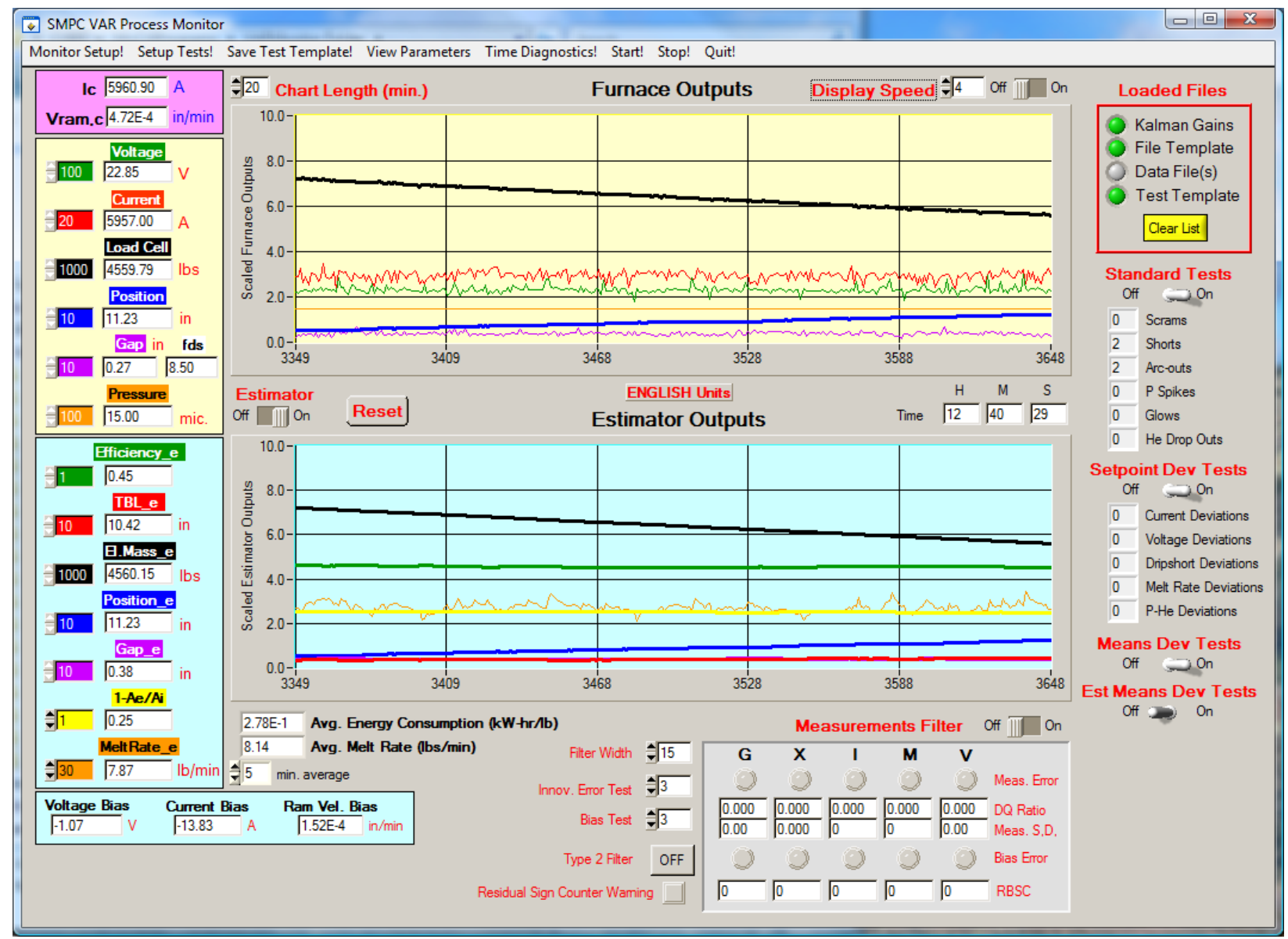

Figure 1. Main operating window for the SMPC VAR monitor.

The VAR and ESR process monitors do not have to be connected directly to a furnace and run in live mode. They may be run using archived computer data files. Up to 1000 data sets may be run simultaneously. When more than one data set is run, the main window shown in Figure 1 is not active. In its place, a popup window appears showing the name of the data set currently being processed. Report files are generated for each data set run. Note that in post-process mode, the monitor may be run and all reports generated with a single mouse click. 
If the VAR or ESR monitor is run in live mode, provision must be made to acquire furnace data. Typically this is done over some type of serial interface between the furnace PLC and the host computer for the monitor, e.g. an Ethernet connection. Data may also be acquired directly by the monitor host computer from the furnace over an A/D converter.

The VAR and ESR monitors have proven to be very effective at detecting and logging process upsets and anomalous melt events both in live mode as well as in post-processing mode. Though this alone does not assure ingot quality, it does help establish process consistency and detect problems early in the processing stream. Because of this, this technology has proven an effective quality tool.

\section{SMPC Process Controllers}

SMPC VAR controller work has resulted in an advanced, model-based controller platform that has three unique modes of control: 1) dynamic melt rate control; 2) ingot input power control; and 3) ingot pool depth control. The first mode accurately controls the melt rate of the electrode (with or without electrode weight feedback) during both steady-state melting and highly transient melting conditions (e.g. during startup, hot-top, or common process upsets like those associated with electrode cracks). The second mode controls the total input power entering the top surface of the ingot consisting of the sum of the electrical power from the arc and thermal power contained as enthalpy in the molten metal dripping from the electrode. This method has proven superior to melt rate control with respect to minimizing perturbations to the solidification zone of the ingot where melt related defects (e.g. freckles in nickel-base alloys) originate. Finally, the third mode involves direct control of the depth of the molten pool atop the ingot. In its present form, this mode uses a linear, low-order VAR process model to make ingot pool depth predictions. The SMPC controller allows for operation in any of these modes, and also allows switching back and forth between modes during melting. Each of the three modes will now be discussed. Note that, as of this writing, only the first mode of operation (melt rate control) has been developed for ESR.

\section{Dynamic Melt Rate Control}

The rate at which the electrode is melted is an extremely important process parameter in both VAR and ESR. Variations cause transients in the ingot growth rate and mushy zone thermal gradient, a condition conducive to the formation of melt related defects [8]. For example, such transients have been linked to freckle formation [9] in nickel-base superalloys, as well as solidification white spot formation in Alloy 718 [10]. It is thought that controlling melt rate during transient melting and through common melt rate disturbances could lead to significant improvements in product yields as well as reduce the number of melt related defects in segregation sensitive alloys [11]. Melt rate control involves controlling electrode gap (or immersion depth) and electrode melting rate by allowing the process current to be whatever it needs to be in order to meet the melt rate reference.

Under normal, steady-state conditions, a reasonably constant melt rate is produced by applying constant melting power. However, this is not the case during startup and end melting. During the initial stages of the remelting process, the temperature distribution in the electrode has not had sufficient time to achieve steady-state. As a result, melt rate continually changes under constant 
power conditions until a steady-state temperature distribution is achieved in the electrode. Toward the end of melting when the melt zone approaches the end of the electrode, heat conduction is impeded because of the large difference in electrode and stub diameters. This causes heat to build up in the electrode end, driving the temperature distribution and, therefore, the melt rate, away from steady-state. In either case, a non-steady temperature distribution in the electrode tip leads directly to a non-steady melt rate at constant power.

Of course, one does not want steady melt rates at the beginning and end of the process. During startup, current is ramped up after an initial hold period, each shop having its own proprietary ramping procedure. Similarly, near the end of melting, a current ramp down schedule (or hot-top procedure) is employed to eliminate or minimize shrinkage cavities in the end of the solidifying ingot. Both startup and hot-top procedures can be relatively complicated, employing multiple current ramps and holds. Given the preceding discussion, it is not surprising that the electrode melt rate does not linearly track the current during these procedures because of startup and end effects. If one wishes to accurately control melt rate during startup and hot-top, the melt rate controller must be capable of implementing dynamic, non-linear current schedules to account for the rapidly changing thermal conditions in the electrode tip.

Transients in the electrode temperature distribution can occur during constant power melting in the absence of startup and end effects for a variety of reasons. An extremely dynamic situation arises when melting through a transverse crack in the electrode. The crack impedes heat flow causing material below the crack to heat up more rapidly than normal while material above the crack remains relatively cold. Under constant power conditions, this leads to increasing melt rate as the melt zone approaches the crack, followed by a rapid decrease as the melt zone passes through the crack. Crack disturbances can last an hour or more in standard size superalloy melts and are unpredictable. They are sometimes referred to as "melt rate events" in industry.

The SMPC has developed and tested dynamic melt rate controllers for both the VAR and the ESR processes. The term "dynamic" is used to indicate that these controllers can "keep up" with highly dynamic melting situations such as those encountered during startup and hot-top, and while melting cracked electrodes. This is accomplished by continually estimating the thermal distribution in the electrode and using process efficiency as a disturbance variable as described in the references. The layout for the nonlinear VAR melt rate controller is shown in Figure 2 [12]. Definitions for the nonlinear functions are given in the reference. Figure 3 shows controller performance while melting through a cracked electrode [13]. The alloy used in this test was a nickel-base superalloy; its exact composition and the conditions under which it was melted cannot be specified due to the proprietary nature of the test. Note the large current oscillations required to hold the melt rate at its setpoint reference. Each oscillation marks a crack in the electrode. Dynamic melt rate control for both VAR and ESR is protected under U.S. Patent $6,115,404$. 


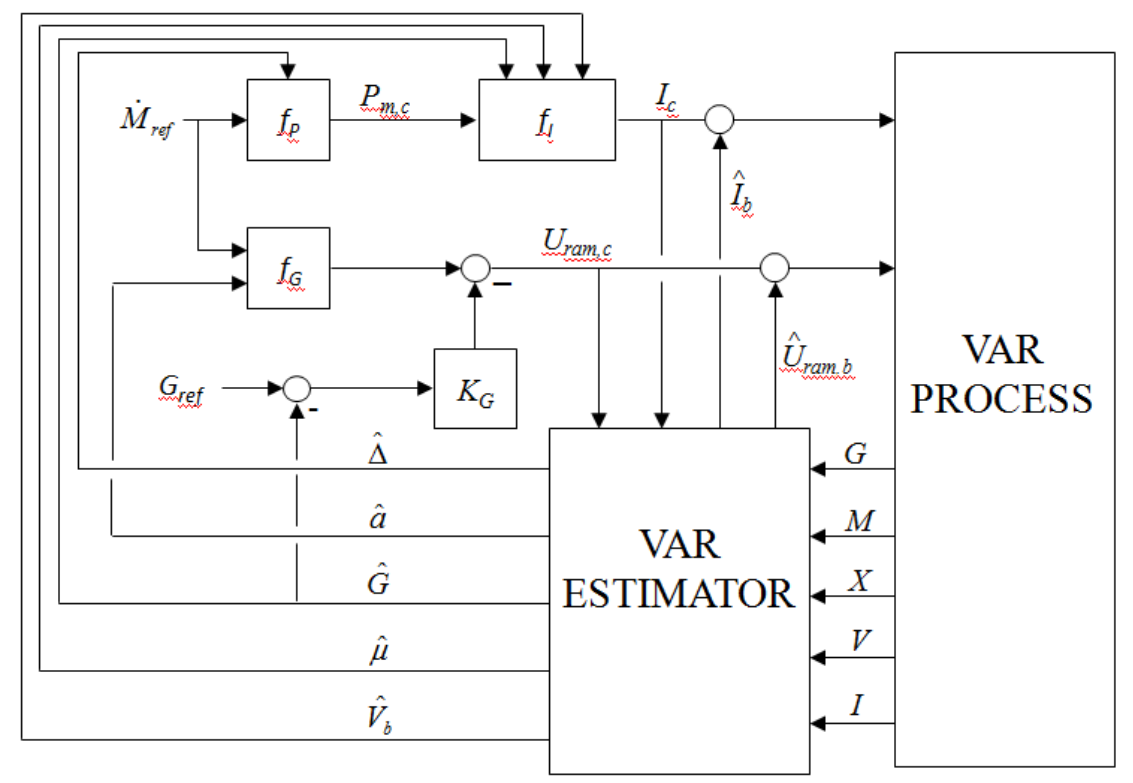

Figure 2. Schematic layout of the nonlinear VAR melt rate controller.

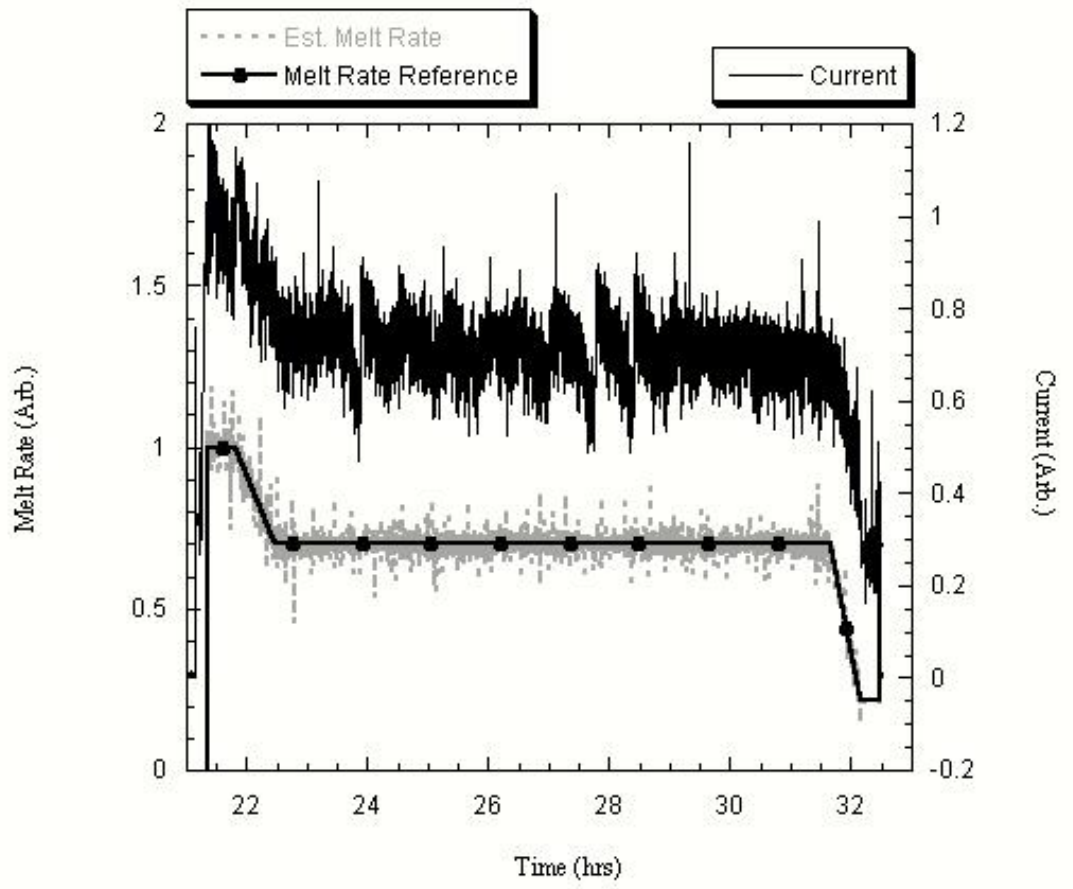

Figure 3. A graph showing the commanded current, melt rate setpoint and estimated melt rate while remelting a cracked superalloy electrode. 


\section{$\underline{\text { Ingot Input Power Control }}$}

It was noted in the last section that relatively large current excursions are required to hold the melt rate constant through crack events. These excursions can give rise to ingot property variations as large as those introduced by melt rate excursions [14]. Hence, melt rate control has no inherent advantage over controlling at constant current under these conditions. It would be better from an ingot quality point of view to allow both the melt rate and current to vary in such a way that the total power entering the top ingot surface is controlled to a constant setpoint value. This is ingot input power control, sometimes called pool power control. The SMPC has developed and tested an ingot input power control mode for VAR and some information is available in the literature [14]. Development work on ESR pool power control has not yet begun.

The layout for the VAR ingot input power controller is shown in Figure 4 [15]. This is a nonlinear controller and the VAR Estimator is mathematically identical to that used in the melt rate controller. The two functions $f_{I}$ and $f_{G}$ are given Reference 14. This controller has been successfully tested on a laboratory scale VAR furnace melting $150 \mathrm{~mm}$ diameter 304SS and Alloy 718 electrodes into $200 \mathrm{~mm}$ ingots. It has also been successfully tested on an industrial furnace melting $430 \mathrm{~mm}$ diameter superalloy electrodes into $510 \mathrm{~mm}$ diameter ingots. The details of the industrial tests are consider proprietary to the SMPC and will not be discussed. Suffice it to say that the controller performed as expected when melting through cracks. A patent application has been submitted to the U.S. Patent Office to protect this technology.

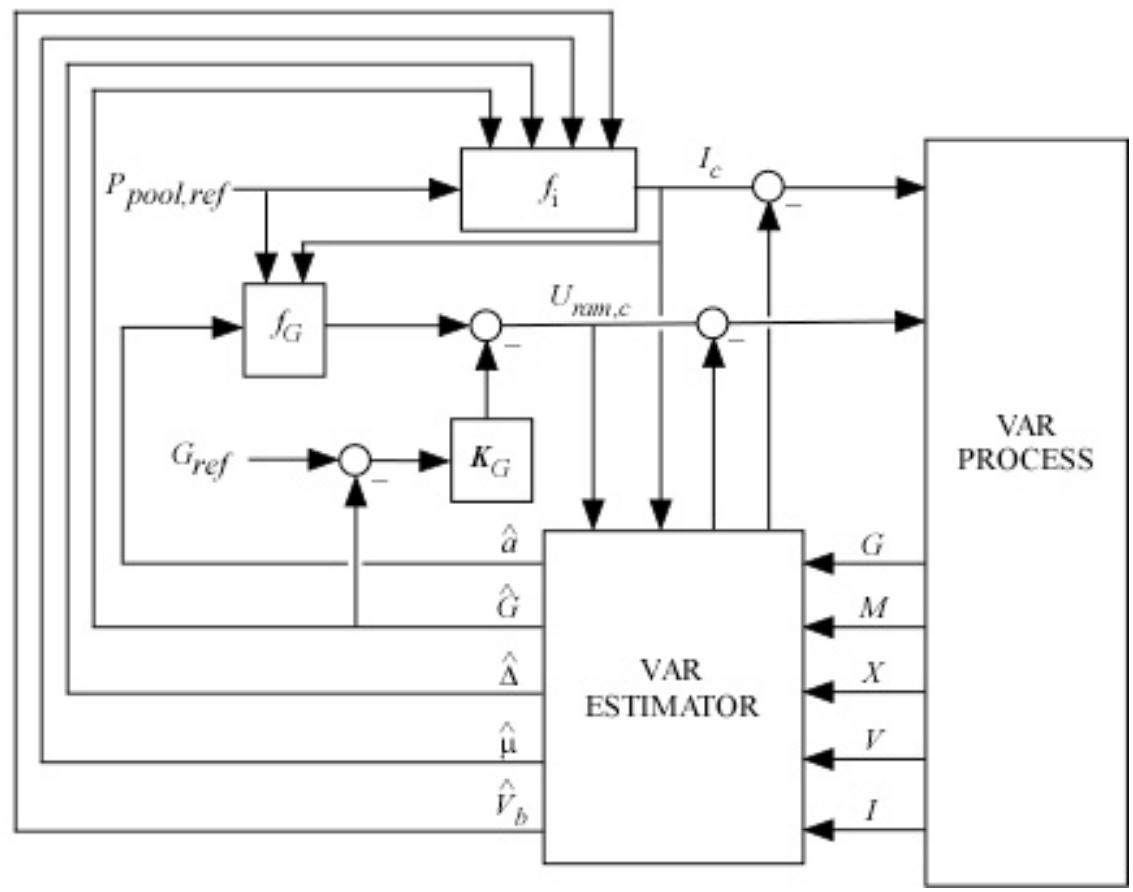

Figure 4. Schematic layout for the SMPC ingot input power controller. 


\section{$\underline{\text { Ingot Pool Depth Control }}$}

VAR ingot pool depth control is the newest control technology developed and tested by the SMPC. Work has not yet begun on the ESR version. The goal is to control the shape of the liquid pool atop the growing ingot during both steady-state and transient melting conditions. Though this is an extremely difficult problem, the payoff for success is very high. If the pool depth can be precisely and stably controlled, the goal of producing material free of defects formed because of unstable, uncontrolled solidification will have been virtually attained. This may enable production of very large diameter superalloy ingots because deep pools are very sensitive to process transients that perturb pool shape and give rise to freckling in these materials.

In its current configuration, the pool depth controller uses the same VAR Estimator used in the other controllers to estimate electrode gap, electrode thermal boundary layer, and process efficiency for feedback to the controller. Additionally, the controller uses a low-order ingot model to estimate pool depth at one or more locations along the ingot radius. Specific information about the ingot model is not available for publication. The open-loop version of the controller has been successfully tested on a laboratory scale VAR furnace melting $150 \mathrm{~mm}$ diameter Alloy 718 electrodes into $200 \mathrm{~mm}$ diameter ingots. A closed loop version of the controller is under development. Because of the proprietary nature of this work, no further details of this controller will be given. A patent application has been submitted to the U.S. Patent Office to protect this technology.

\section{Summary}

Over the past few years, the SMPC has developed VAR and ESR process monitors and controllers in an effort to improve ingot quality and work toward the goal of eliminating melt related defects in ingots. The process monitors use Kalman filter technology to provide estimates for process variables that can be compared with measurements to detect process upsets. Conventional input data tests are also used to detect and flag process upsets and events that may give rise to melt related ingot defects. The process estimators used in these monitors have been incorporated into VAR and ESR melt rate controllers. This technology allows precise control of melt rate during highly transient conditions such as encountered during startup, hot-top, or when remelting cracked electrodes.

In addition to highly dynamic melt rate control, ingot input power control has been developed and tested for the VAR process. This control technology allows direct control of the total power entering into the ingot pool surface from both the arc and the molten metal enthalpy. This type of control has been shown to be more effective at minimizing the perturbations to the solidification zone during a crack event that either constant current control or constant melt rate control.

Finally, an open loop ingot pool depth controller has been developed and tested on a laboratory scale furnace during VAR of Alloy 718. This control technology is in its infancy but as more effective and advanced forms of pool depth control are developed it will allow for direct control of the solidification zone in the ingot, a capability that will allow melt engineers to directly manipulate and evaluate those ingot conditions that give rise to the formation of melt related defects. 


\section{References}

1. Current SMPC members: ATI Allvac, Carpenter Technology, Special Metals, Titanium Metallurgical (Timet), and Rolls-Royce.

2. B. Friedland, Control System Design, An Introduction To State-Space Methods, (New York, NY: McGraw-Hill, 1986), p. 259.

3. R.L. Williamson, J.J. Beaman, D.K. Melgaard, G.J. Shelmidine, and R. Morrison, "ModelBased Melt Rate Control during Vacuum Arc Remelting of Alloy 718," Metall. And Mat. Trans. $B, 35 B,(2004), 101-13$.

4. D.K. Melgaard, G. Shelmidine and J.J. Beaman, "Multiple-Input Multiple-Output Electroslag Remelting Controller for Coupled, Consistent melt Rate and Immersion Depth Control," Proc. of The 2005 Int'l Symposium on Liquid Metal Processing And Casting, ed.'s P.D. Lee, A. Mitchell, R.L. Williamson and S. Ballantyne (Materials Park, OH: ASM International, 2005), 219-226.

5. J.J. Beaman, R.L. Williamson and D.K. Melgaard, "A Nonlinear Reduced Order Model for Dynamic VAR Melt Rate Control," Proc. of The 2001 Int'l Symposium on Liquid Metal Processing And Casting, ed.'s A. Mitchell and J. Van Den Avyle (New York, NY: American Vacuum Society, 2001), 161-174. See also Reference 3.

6. R. L. Williamson, R. G. Erdmann, J. J. Beaman and D. K. Melgaard, "Monitoring the Vacuum Arc Remelting Process," Proc. of The 2007 Int'l Symposium on Liquid Metal Processing And Casting, ed.'s P. D. Lee, A. Mitchell, J.-P. Bellow and A. Jardy (Paris, France: Société Française de Métallurgie et des Matériaux, 2007), 1-6.

7. SMPC VAR and ESR monitor software packages were developed in the C programming language using the LabWindows CVI programming environment from National Instruments, Inc., Austin, Texas.

8. M.C. Flemings, Solidification Processing (New York, NY: McGraw-Hill, 1974), 245.

9. T. Suzuki, T. Shibata, K. Morita, T. Taketsuru, D.G. Evans and W. Yang, Proc. of The 2001 Int'l Symposium on Liquid Metal Processing And Casting, ed.'s A. Mitchell and J. Van Den Avyle (New York, NY: American Vacuum Society, 2001), 325-337.

10. L.A. Bertram, J.A. Brooks, D.G. Evans, A.D. Patel, J.A. Van Den Avyle and D.D. Wegman, Proc. of The 1999 Int'l Symposium on Liquid Metal Processing And Casting, ed.'s A. Mitchell, L. Ridgway and M. Baldwin, (New York, NY: American Vacuum Society, 1999), 156-167.

11. R.L. Williamson, J.J. Beaman, D.K. Melgaard, G.J. Shelmidine, A.D. Patel, and C.B. Adasczik, "A Demonstration of Melt Rate Control during VAR of Cracked Electrodes," J. Mat. Sci., 39 (2004), 7161-7168. 
12. Figure taken from J.J. Beaman, R. L. Williamson, D. K. Melgaard, G.J. Shelmidine, and J.C. Hamel, "Model Based Gap and Melt Rate Control for VAR of Ti-6Al-4V," Proc. of The 2003 Int'l Symposium on Liquid Metal Processing And Casting, ed.'s P.D. Lee, A. Mitchell, J.-P. Bellot and A. Jardy (Paris, France: Société Française de Métallurgie et des Matériaux, 2003), $67-$ 76.

13. Figure taken from Reference 3.

14. J.J. Beaman, R.L. Williamson, D.K. Melgaard, R.M. Aikin, Jr., and R.G. Erdmann, "Vacuum Arc Remelting Pool Power Control," Proc. of The 2007 Int'l Symposium on Liquid Metal Processing And Casting, ed.'s P. D. Lee, A. Mitchell, J.-P. Bellow and A. Jardy (Paris, France: Société Française de Métallurgie et des Matériaux, 2007), 37-42.

15. Figure taken from Reference 14. 